\title{
High Field MRI in Human African Trypanosomiasis (HAT)
}

\section{Cherif Mohamadou Aidara ${ }^{1}$, Caroline Magne ${ }^{2}$, Philomene Kouna ${ }^{2}$, Gaelle Ebinda Mipinda ${ }^{3}$, Abdoulaye Dione Diop', Abdoulaye Ndoye Diop', Sokhna Ba ${ }^{1}$}

\author{
${ }^{1}$ Radiology Office of Fann University Hospital, Dakar-Fann, Sénégal \\ ${ }^{2}$ Internal Medecine Office of Polyclinique El Rapha Trois Quartiers, Libreville, Gabon \\ ${ }^{3}$ Radiology Office of Polyclinique El Rapha Trois Quartiers, Libreville, Gabon \\ Email:matoouz@gmail.com
}

How to cite this paper: Aidara, C.M., Magne, C., Kouna, P., Mipinda, G.E., Diop, A.D., Diop, A.N. and Ba, S. (2017) High Field MRI in Human African Trypanosomiasis (HAT). Open Journal of Radiology, 7, 190-198.

https://doi.org/10.4236/ojrad.2017.73021

Received: June 29, 2017

Accepted: September 4, 2017

Published: September 7, 2017

Copyright $\odot 2017$ by authors and Scientific Research Publishing Inc. This work is licensed under the Creative Commons Attribution International License (CC BY 4.0).

http://creativecommons.org/licenses/by/4.0/

\begin{abstract}
Human African Trypanosomiasis (HAT) or sleeping thickness is a forest and rural disease; where agriculture is the main activity. It is a chronic and lethal disease without treatment. HAT is caused by two parasites; Trypanosoma Brucei Gambiense (gTB) and Trypanosoma Brucei Rhodesiense (rTB) transmitted to humans by the tsetse fly. It is endemic condition in Africa between the $15^{\circ}$ north latitude and the $20^{\circ}$ south latitude. It is reported outside this area in travelers who stayed in endemic zone. Infection by gTB is wider and more frequent (98\%) than that by rTB (2\%). The Democratic Republic of Congo is the most affected country with more than $75 \%$ of reported cases. The geographical distribution is not homogeneous. There are more affected regions in a zone called "foci" which represents areas favorable to the development of the vector. Its diagnosis and treatment are very important because of its social and economic impact at both the individual and community levels. Promising molecules including fexinidazole are currently undergoing testing. Nowadays populations move more and more easily but the discovery of this disease in daily neuroradiological practice is exceptional. We propose in this paper through two observations, reminders on epidemiological, clinical and MRI features of HAT. It typically performs the edematous, bilateral and diffuse encephalitis. It is important to distinguish these aspects from the arsenic-induced encephalitis that may occur during treatment. Only vector control allows eradicating this disease. WHO has set targets elimination of HAT as a public health problem for 2020 deadline.
\end{abstract}

\section{Keywords}

Human African Trypanosomiasis, High Field MRI, Diffuse Encephalitis, Optic Tract, Arsenic-Induced Encephalitis 


\section{Introduction}

Human African Trypanosomiasis (HAT) is an anthropozoonosis disease due to two flagellated protozoan species; Trypanosoma Brucei Gambiense (gTB) and Trypanosoma Brucei Rhodesiense (rTB) transmitted to humans by the tsetse flies or glossina. It is an endemic condition between the $15^{\circ}$ north latitude and the $20^{\circ}$ south latitude, a favorable area to development of the vector. So it is endemic in Central, Eastern and Western Africa. It's a lethal condition without treatment. To date there is not effective preventive therapy.

Only anti-vector control can eradicate the disease. Under control in the 1960s, it re-emerged in the mid of 1990s. Government, non-governmental and WHO programs are working together to eradicate it. Diagnostic techniques have been improved significantly (PCR) but remain expensive in our regions where national health programs are sometimes faulty and difficult to use on a large scale. The detection of serum IgM by CATT and the search for trypanosome in the CSF are the most commonly used techniques. Treatment is currently based on protocols combining pentamidine, effornithin, nifurtimox and melarsoprol.

Its features in MR imaging are poorly reported in endemic areas. People move better than the past and cities have a higher technical medical level than rural areas. We illustrate through these two observation reminders on epidemiological, clinical, MRI features and the treatment of this condition.

\section{Case Presentation}

\subsection{Observation 1}

\subsubsection{Clinical Features}

Ms OH C 40 years was addressed in MRI for headache associated with alertness disorder. Nocturnal insomnia and diurnal somnolence, left hemiparesis and extrapyramidal syndrome were noted and a slight fever. Brain CT showed diffuse hypodensity on the central gray nuclei without significant contrast enhancement. MRI was performed on a high-field device $1.5 \mathrm{~T}$ siemens in the 3 planes with T1 SE, T2 SE, T2 FLAIR, T2* and T1 gadolinium sequences.

\subsubsection{Biochemical Examinations}

The biological examinations showed a biological inflammatory syndrome with elevation of CRP without abnormality on the blood. Serum IgM testing by CATT showed positivity; the lumbar puncture detected trypanosomes in the CSF.

\subsubsection{MRI Fearures}

MRI showed predominant vasogenic cerebral edema on the gray nuclei and the right semi-oval center. Small focus of cytotoxic edema on the right internal capsule. Achievement of the thickened and hypersignal right optical strip. Minimal contrast. Discreet mass effect on the right lateral ventricle dispite the extending edema (Figures 1-3). 

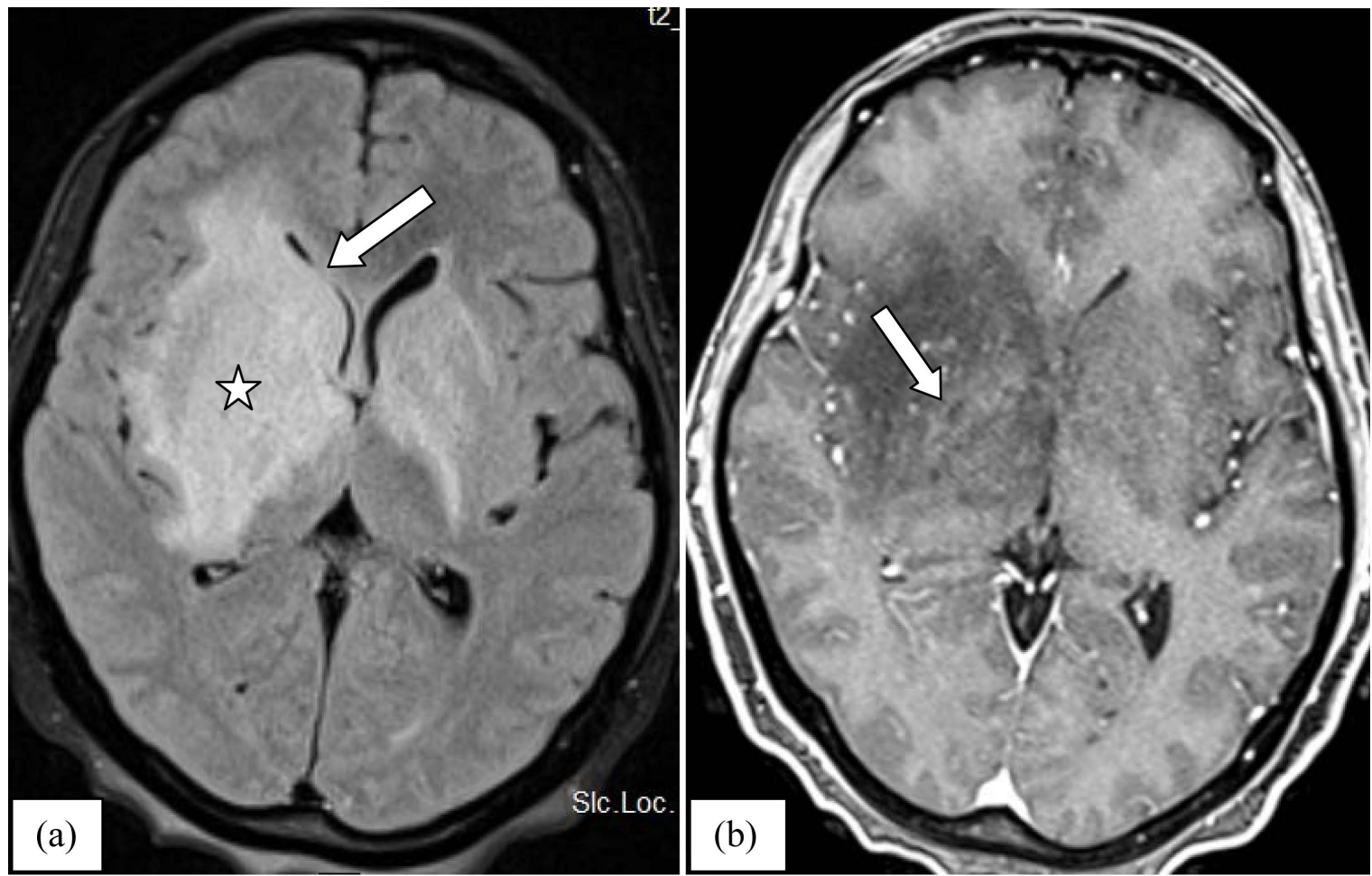

Figure 1. MRI axial section T2 FLAIR on (a), and a T1 weighted after gadolinium on (b). Extended edema on the basal ganglia and the white matter predominantly on the right side (star (a)). Moderate mass effect on the right lateral ventricle (arrow in (a)) despite the extended edema. Very slight contrast enhancement in the posterior arm of the internal capsula $(\operatorname{arrow}(b))$.
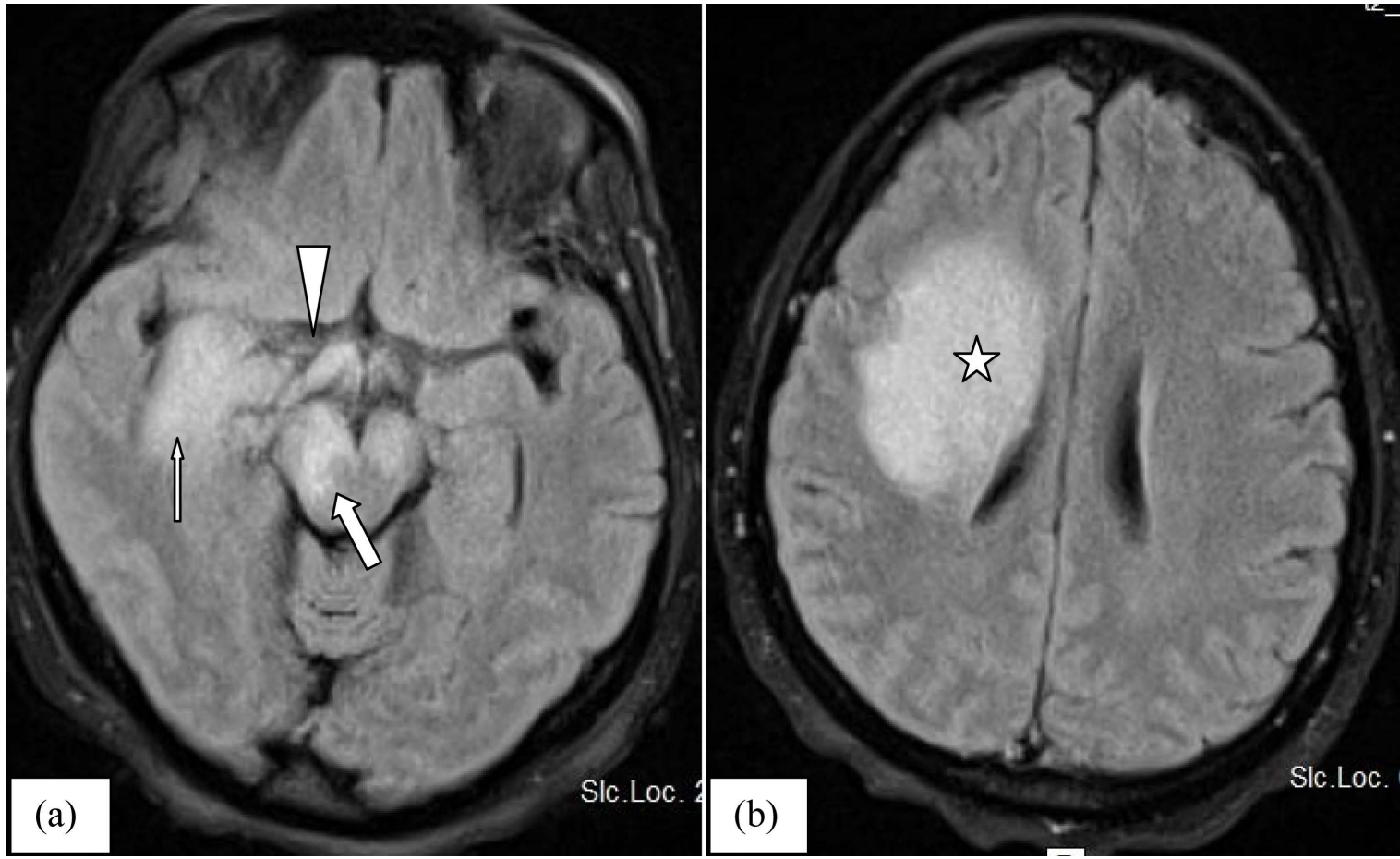

Figure 2. MRI axial section weighted T2 FLAIR in (a) and (b) showing the vasogenic edema extending upwards on the internal temporal region (thin arrow on (a)), the frontal white matter (star on (b)), the peduncle (thick arrow on (a)) and the right optic tract (arrow head in (a)). 


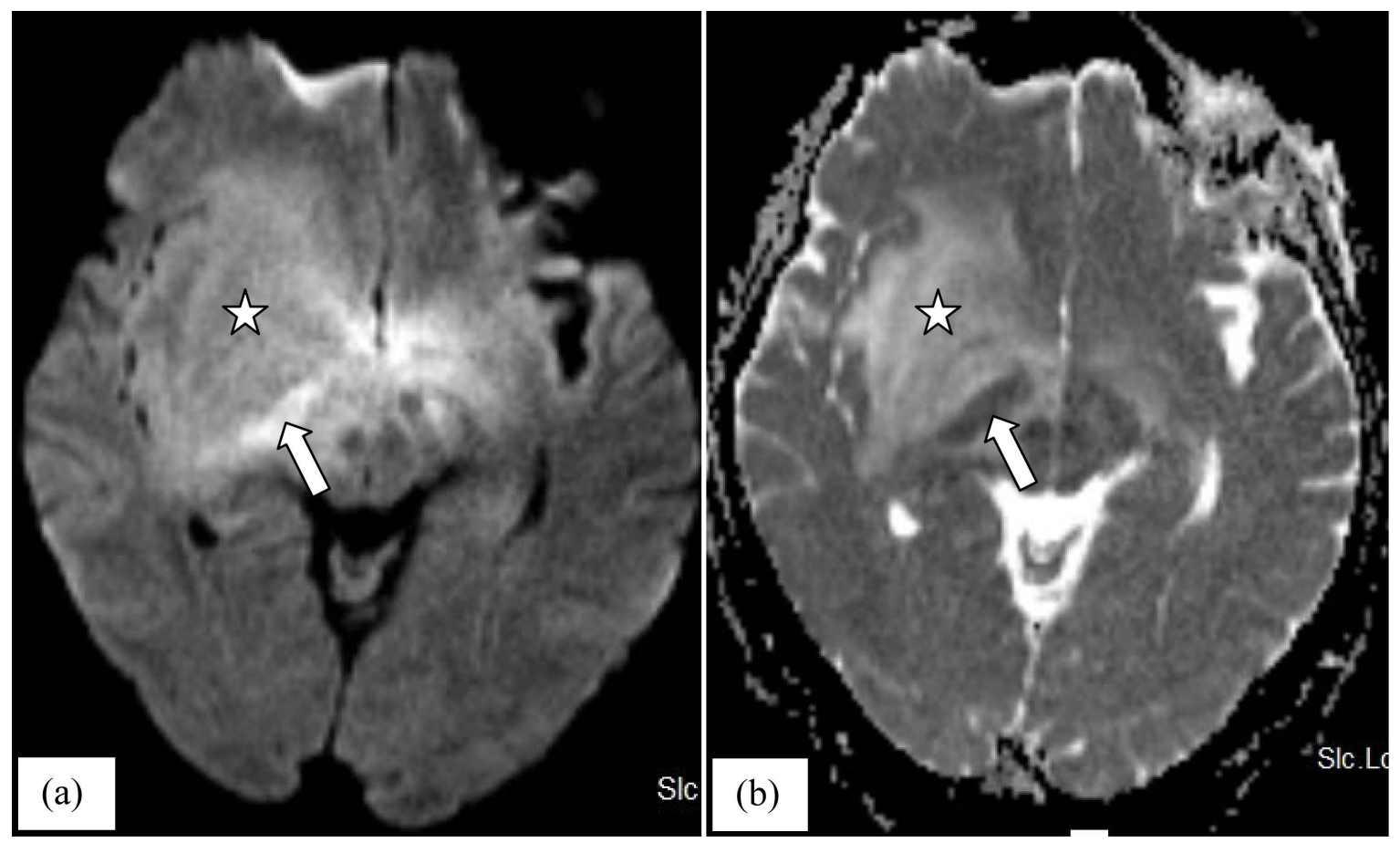

Figure 3. MRI axial section diffusion weighted B1000 on (a) showing a focus restriction area hyperintense on the posterior arm of the internal capsula (arrow). Restriction on the ADC map on (b) hyposignal (arrow in (b)). Vasogenic edema predominates elsewhere in the basal ganglia and the white matter (star on (a) and (b)).

\subsubsection{Evidence Diagnostic}

Meningo encephalitis stage of HAT.

\subsubsection{Treatment}

The treatment was admistered in the trypanosomiasis specialized center and was based on pentamidine $200 \mathrm{mg} /$ day intramuscular injection associated with arsobal $180 \mathrm{mg} /$ day, dexamethasone $24 \mathrm{mg} /$ day and ampicillin $4000 \mathrm{~g} / \mathrm{day}$. Administration of serum glucose 1.5 to $2 \mathrm{~L}$ /day with addition of $\mathrm{NaCl}, \mathrm{KCl}$ and multivitamin complex.

\subsection{Observation 2}

\subsubsection{Clinical Features}

Mr M K, 45 years old, chronic ethyl soil, was admitted to the emergency room for alteration of consciousness associated with a tremor. Fever was not noted. Abnormal somnolence has proceeded about a month. MRI scan was done using the same protocole.

\subsubsection{Biochemical Examination}

They had shown an inflammatory syndrome with elevated CRP, a slight microcytosis without anemia and a slight neutrocytosis. Detection of serum IgM anti-trypanosomes by CATT and the search for trypanosomes on the CSF was positive.

The lumbar puncture showed hyperproteinorachia, hypoglycorachia and hyperchlorurorachia. 


\subsubsection{MRI Features}

MRI showed bilateral vasogenic diffuse edema on the gray nuclei, semi-oval centers, protuberance and cerebral peduncle. Reaching of the right optical tract was noted. Little mass effect. No haemorrhage or significant contrast enhancement were noted (Figure 4 and Figure 5).
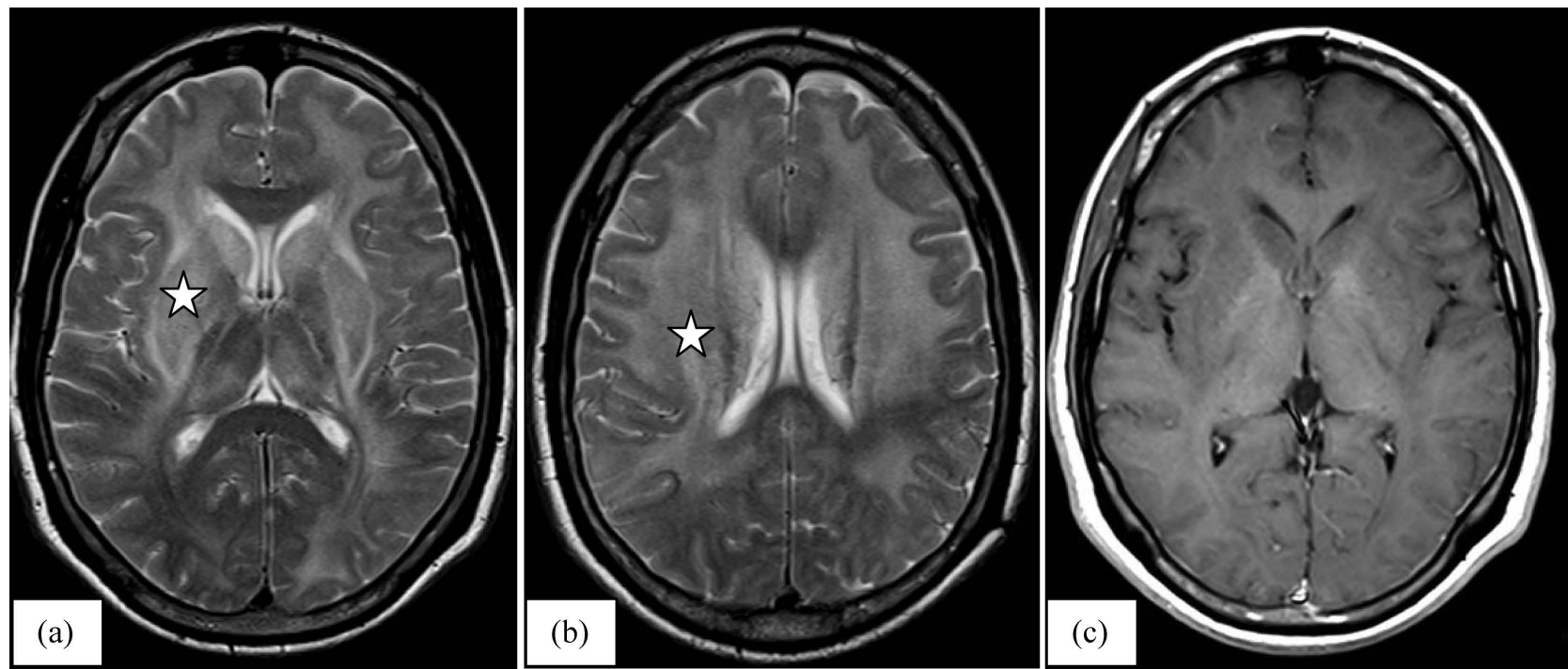

Figure 4. MRI axial sections T2 weighted ((a) and (b)) showing a diffuse edema of the white matter of the semi-oval centers and the basal ganglia (star (b)) without any significant contrast enhancement in the T1 weighted axial section on (c).
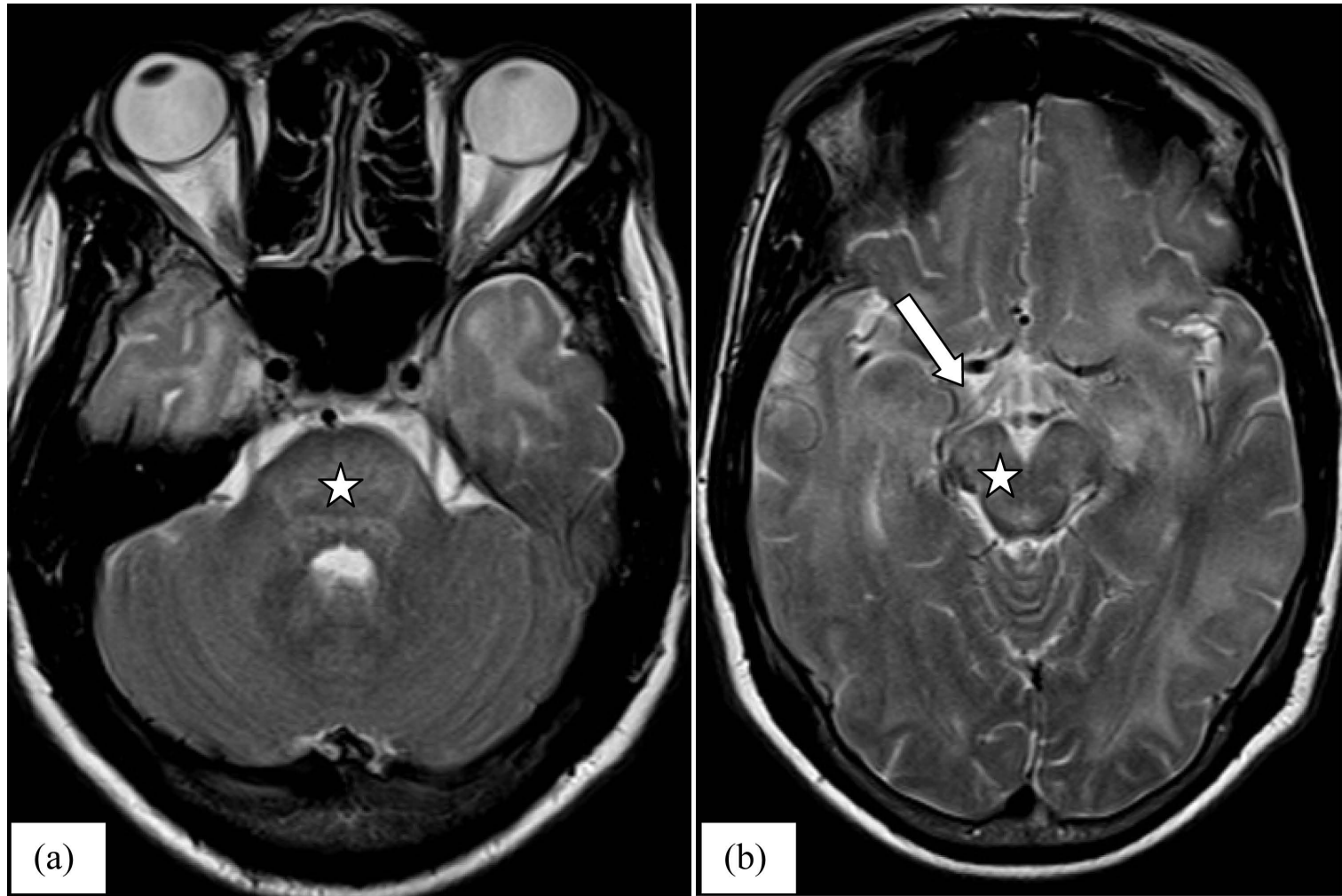

Figure 5. MRI T2 weighted axial section on (a) and (b) showing the extended edema to the brain stem (stars) and reaching the right optic tract (arrow (b)). 


\subsubsection{Evidence Diagnostic}

Encephalitic stage of HAT.

\subsubsection{Treatment}

The same protocole was performed at the trypanosomiasis specialized center.

\section{Commentary}

\subsection{HAT Epidemiology}

HAT is endemic in 36 countries in sub-Saharan Africa corresponding to an exposure of 50 to 60 million inhabitants. Tropical disease once neglected, it now forms part of the WHO roadmap for diseases to be eradicated by 2020 as a public health problem. In 2012 the number of new cases registered was less than 8000 [1] [2] [3]. gTB is prevalent in Western and Central Africa while rTB is prevalent in Eastern and Southern Africa. This condition occurs in areas of high vegetation which maintains moisture, temperature and luminosity favorable to the development of its vector, the tsetse fly or glossina. The difficult access to rural areas and the urbanization of cities make this disease an exceptional discovery in current neuroradiological practice. People are moving now better than the past. Its features in imaging, particularly in high field MRI must be known in order to launch the etiological investigation as quickly as possible.

\subsubsection{What Are the Clinical Features of HAT?}

Natural history of HAT is classically divided into 3 phases. The inoculation stage induced by the bite of the tsetse fly causes a local inflammatory reaction called trypanoma. It leads to focal induration with erythema, local elevation of temperature and hyperaesthesia. After regression about 3 weeks, it leaves a hyperpigmented scar that can last several years.

The haemolymphatic stage is characterized by the importance of general signs with fever, lymphadenopathy, generalized pruritus, rash, edema, anemia, cardiovascular and endocrine disorders, renal involvement and infections. Mainly, pulmonary infection can cause death.

The encephalic stage is characterized by disorders of alertness, sleep disorder with diurnal somnolence and night insomnia, leading to permanent hypersomnia and coma, which are the main characteristic of this HAT. This desease is also called "sleeping sickness". Other abnormalities of the nervous system are also noted; motor and tonus disorders, modification of archaic osteo-tendinous and cutaneous reflexes, sensory disturbances with cutaneous and deep hyperaesthesia, psychiatric disorders, electrogenesis disorders [2] [4] [5].

\subsubsection{Patho-Physiology and Imaging Aspects of HAT}

The parasite gets into the nervous system through the leptomeninge, reaching the sub-arachnoid space where it becomes detectable in the CSF. It finaly gets in the brain tissue through the Virchow-Robin spaces [6]. It induces a perivascular inflammatory reaction. If CT is normal at this stage, MRI may show meningeal or parenchymal abnormalities. In addition, MRI can differentiate between the lesion 
induced by the parasite and that due to melarsopol induced-encephalitis. Arsenical derivative forming part of the therapeutic arsenal of encephalitic form [2] [6].

The HAT performs focal lesions, typically bilateral and quite symmetrical, on the grey nuclei, the cerebellum and the splenium of the corpus callosum [5] [6]. Later, diffuse hyperintensity is observed on the internal capsula, the external and extreme capsula associated with an attack of the cortex and deep grey nuclei. These aspects were noted in our two patients but without involvement of the cortex or the corpus callosum. The involvement of the optical pathways and the brainstem seems to be caracteristical (Figure 1 and Figure 2). In the case reported by Sabbah there was a meningeal or medium cerebellar peduncle lesion initially. There is no gadolinium enhancement. In our patients, signal of the lesions was predominantly a vasogenic edematous type; hyposignal T1, hypersignal T2, without zone of significant restriction. In the patient of the observation 1 , the small restriction zone on the posterior arm of the internal capsula explained the partial deficit clinically described. It's a singular remark that there was no focus haemorrhage.

Through our two observations and the cases reported in the literature, the typical aspects of HAT result in bilateral and quasi-symmetrical vasogenic edema involving the basal ganglia, the white matter, the brainstem. There is no significant contrast enhancement or mass effect despite the extending edema. Reaching of the visual pathway, the optic tract particularly seems characteristic in our 2 observations (Figure 2(a) and Figure 5(b)). We have not found in the rewiew of the litterature involvement of the optic tract in patient suffering from HAT. However in the case reported by Gill et al. involvement of the optic radiation have been discribed [6] [7] [8].

These topographic and signal features of the HAT make possible to evoke the diagnosis before launching aetilogical research. MRI also make possible to distinguish lesions due to HAT from an arsenical encephalitis induced by melarsoprol administered during the treatment. It is a serious complication since it is fatal to $5 \%$ and whose survival is burdened with heavy sequelae. Checkley et al. reported a case of survival at the cost of a locked-in syndrome. Arsenical encephalitis typically results in haemorrhagic lesions with severe edema [5] [7] [9].

\subsection{Anatomopathological Aspects}

Lesions of HAT correspond to a perivascular sleeve made of lymphocytes both in the parenchyma and in the arachnoid. The vessels are surrounded by demyelination foci and axonal damage in which microglial cells and gemocytes are found. These lesions predominate in the white matter and without any focus haemorrage [5].

\subsection{Evidence Diagnosis}

Evidence diagnosis is based on finding the trypanosome in the trypanoma at the inoculation phase, in the blood or the ganglionic juice at the lymphatic-sanguine phase or in the CSF in the meningoencephalitis phase. 
Serological diagnosis is also possible in the blood or CSF by detection of anti-trypanosome antibodies IgM [2] [4] [10]. PCR techniques are also available and remain more efficient. Its use on a large scale should make possible to detect the disease early and also allows differentiating the parasites rTB and gTB [3].

\subsection{Treatment}

Curative treatment is based on pentamidine, eflornithine, nifurtimax and melarsoprol for the gTB form. The rTB form is treated with suramine and melarsoprol. Currently trials are underway for treatment with fexinidazole [1] [2] [3] [4] [9] [10] [11].

Melarsoprol is a toxic arsenical derivative responsible for encephalopathy that may occur in $10 \%$. Its mortality is estimated between $3 \%$ to $5 \%$ [9]. Relapse is not negligible estimated in $20 \%$ in Robays' study [10].

There is no available preventive treatment for this fatal disease. These aspects highlight the importance of anti-vector treatment as the only guarantee of efficacy for the eradication of HAT [1] [2] [3].

\section{Conclusion}

The management of trypanosomiasis from diagnosis to treatment, rests mainly on the responsibilities of specialized centers. Wider use of diagnostic tools, should be extended to any medical structure for wider sentinel oversight. On the other hand, imaging, MRI particularly, must be integrated into the tools of care and follow-up of the patients both to guide the etiological research and to seek the complications related to the melarsoprol-based treatment.

\section{References}

[1] WHO Trypanosomiasis Human African http://www.who.int/mediacentre/factsheets/fs259/en/

[2] Braakman, H.M.H., Van de Molengraft, F.J.J.M., Hubert, W.W.A. and Boerman, D.H. (2006) Lethal African Trypanosomiasis in a Traveler: MRI and Neuropathology. Neurology, 66, 1094-1096. https://doi.org/10.1212/01.wnl.0000209306.41647.13

[3] Amongi, C. (2014) Epidemiology and Control of Human African Trypanosomiasis in Uganda. https://www.era.lib.ed.ac.uk/handle/1842/16246

[4] Howlet, W.P. (2012) Protozoal an Helmintic Infections. In: Howlet William, P., Ed., Neurology in Africa 2012 Norway, 156-164.

[5] Sabbah, P., Brosset, C., Imbert, P., Bonardel, G., Jeandel, P. and Briant, J.F. (1997) Human African Trypanosomiasis: MRI. Neuroradiology, 39, 708-710. https://doi.org/10.1007/s002340050491

[6] Gill, D.S., Chatha, D.S. and Carpio-O’Donovan, R.D. (2003) MR Imaging Findings in African Trypanosomiasis. AJNR, 24, 1383-1385.

[7] Kager, P.A., Schipper, H.G., Stam, J. and Majoie, C.B.L.M. (2009) Case Report: Magnetic Resonance Imaging Findings in Human African Trypanosomiasis: A Four-Year Follow up Study in a Patient and Review the Literature. American Journal of Tropical Medicine and Hygiene, 80, 947-952. 
[8] Rodgers, J., Jones, A., Gibaud, S., Bradley, B., McCabe, C., Barrett, M.P., et al. (2011) Melarsoprol Cyclodextrin Inclusion Complexes as Promising Oral Candidates for the Treatment of Human African Trypanosomiasis. PLoS Negl Trop Dis, 5, e1308. https://doi.org/10.1371/journal.pntd.0001308

[9] Checkley, A.M., Pepin, J., Gibson, W.C., Taylor, M.N., Jäger, H.R. and Mabey, D.C. (2007) Human African Trypanosomiasis: Diagnosis, Relapse and Survival after Severe Melarsoprol-Induced Encephalopathy. Tropical Medicine \& Hygiene, 101, 523-526. https://doi.org/10.1016/j.trstmh.2006.12.001

[10] Robays, J., Nyamowala, G., Sese, C., Mesu Kande, V.B.K., Lutumba, P., Van Der Veken, W. and Coll (2008) High Failure Rates of Melarsoprol for Sleeping Sickness, Democratic Republic of Congo. Emerging Infectious Diseases, 14, 966-967. https://doi.org/10.3201/eid1406.071266

[11] Giordani, F., Mwenechanya, R. and Barrett, M. (2014) Advances in Understanding and Treatment of Human African Trypanosomiasis: Divergent Diseases Caused by Distinct Parasites. In: Padmanabhan, S., Ed., Handbook of Pharmacogenomics and Stratified Medicine, Academic Press, 901-917. https://doi.org/10.1016/B978-0-12-386882-4.00039-6

\section{Submit or recommend next manuscript to SCIRP and we will provide best} service for you:

Accepting pre-submission inquiries through Email, Facebook, LinkedIn, Twitter, etc. A wide selection of journals (inclusive of 9 subjects, more than 200 journals)

Providing 24-hour high-quality service

User-friendly online submission system

Fair and swift peer-review system

Efficient typesetting and proofreading procedure

Display of the result of downloads and visits, as well as the number of cited articles

Maximum dissemination of your research work

Submit your manuscript at: http://papersubmission.scirp.org/

Or contact ojrad@scirp.org 